\title{
武汉市公共交通网络中心性及其与银行网点的 空间耦合性研究
}

\author{
詹 璇 ${ }^{1,2}$, 林爱文 ${ }^{1,2^{*}}$, 孙 铖 ${ }^{1,2}$, 乔 卫 ${ }^{1}$ \\ (1. 武汉大学资源与环境科学学院, 武汉 430079; 2. 教育部地理信息系统重点实验室, 武汉 430079)
}

\begin{abstract}
摘 要: 本文以构建公共交通网络为切人点, 运用改进的多中心性评价模型测度了武汉都市发展区公共交通网络中 心性, 并结合 GIS 核密度分析法与双变量空间自相关分析法, 探讨了公共交通网络中心性和银行网点空间分布规 律, 以及两者之间的耦合性与空间结构。主要研究结论为: 1)武汉都市发展区公共交通网络中心性具有城市多中 心指向性特征, 且由中心向外围圈层递减; (2)银行网点布局呈现“核心一中心一过渡区一外围”多层次结构, 且具有 空间不均衡性; (3)公共交通网络各中心性指标与银行网点具有不同程度的空间正相关。银行网点受接近中心性的 影响最大, 直达性次之, 介数中心性最小; (4)局域上, 公共交通网络中心性指标与银行网点的耦合关系存在空间不 平稳性与空间异质性。高一高聚集与低一低聚集是主要的空间关联模式; 高一高聚集主要位于银行网点布局的核 心圈层,低一低聚集点缀于外围圈层,高一低聚集与低一高聚集介于核心圈层与外围圈层之间。
\end{abstract}

关 键 词: 公共交通网络中心性; 改进多中心性评价模型; 银行网点; 耦合; 武汉都市发展区

\section{1 引言}

城因水兴,市缘路起。城市交通路网是城市发 展和居民生活出行重要的物质基础, 在实现城市日 常功能的同时, 影响着城市的空间结构与发展(黄 文雄等, 2009; 李清泉等, 2012)。公共交通作为城 市交通系统的主体部分, 是解决居民通勤问题、缓 解交通环境压力、改善要素集聚分配的有效手段 (李志等, 2014; 许文娟, 2014)。《中华人民共和国国 民经济和社会发展第十二个五年规划纲要》中提 出, 要大力发展城市公共交通系统, 实施公交优先 发展战略。优先发展城市公共交通已成为缓解交 通拥堵、构建两型社会的必然选择(徐黔予, 2011; 王薇等, 2012)。

1998 年, 复杂网络研究爆发, 许多真实世界的
网络受到社会学、生物学、物理学等领域学者的热 切关注。公共交通系统作为一种具有复杂结构的 现实网络, 国内外众多交通及地理学者从复杂网络 理论的视角对其展开研究(赵莉莉, 2010)。如 Latora 等(2002)初步研究了波士顿地铁的网络特性; Sienkiewicz 等(2005)探讨了波兰 21 个城市的公共 运输网络的聚类系数、介数及匹配性等拓扑特性。 国内公共交通复杂网络的研究起步稍晚。高自友 等(2005)以北京市为例, 对城市公交网络的无标度 特性及度分布指数进行了实证研究; 王喆等(2007) 构建了成都市公交线路系统的复杂网络,通过计算 网络拓扑结构的静态几何量参数来分析其拓扑特 性。以上成果大多是基于复杂网络理论对公交网 络形态结构及整体评价进行的研究,对城市公交网 络空间特性与社会经济活动之间联系的研究较

收稿日期:2016-02;修订日期:2016-07。

基金项目: 国家基础科学人才培养基金项目(J1103409) [Foundation: Fund for Fostering Talents in Basic Science of the National Natural Science Foundation of China, No.J1103409]。

作者简介: 詹璇(1992-), 女, 江西上饶人, 硕士生, 研究方向为人文地理学、城市地理学及 GIS 应用,E-mail: 254298610@qq.com。 通讯作者: 林爱文(1963-), 男, 湖北黄冈人, 教授, 博士生导师, 主要从事区域发展与土地利用、资源环境遥感与 GIS 应用研究, E-mail: awlin@whu.edu.cn。

引用格式:詹璇, 林爱文, 孙铖, 等. 2016. 武汉市公共交通网络中心性及其与银行网点的空间耦合性研究 [J]. 地理科学进展, 35(9): 1155-1166. [Zhan X, Lin A W, Sun C, et al. 2016. Centrality of public transportation network and its coupling with bank branches distribution in Wuhan City[J]. Progress in Geography, 35(9): 1155-1166.]. DOI: 10.18306/dlkxjz.2016.09.010 
少。随着“可达性”这一概念的兴起, 交通可达性也 逐渐受到国内研究者的重视, 并广泛运用于交通系 统与经济发展(程钰等, 2013)、人口变化(李涛等, 2012)、居民出行行为(曹小曙等, 2015)、社会活动空 间关系(李立等, 2012)等方面。公共交通作为交通 系统的一个重要子集, 其可达性研究也随之增多。 如田关云(2014)利用 TransCAD 建立了兰州市公共 交通路线系统,并评价其可达性水平;李志等(2014) 以南京地铁 $1 、 2$ 号线为例, 测度了地铁建成前后的 公共交通可达性及其格局变化。公共交通网络中 心性是测度可达性的一种有效手段,在城市地理、 公共交通规划等理论与实践研究中发挥重要作用, 如李刚(2014)从公共交通网络中心性角度研究了城 市内部轨道交通应急救援站的选址问题。总体而 言, 上述研究成果在一定程度上填补了公共交通网 络与城市社会经济活动关系领域的空缺。

伴随全球化的快速发展, 金融服务业日益成为 各国经济发展中的主动力与创新源泉 (Bryson, 1997)。银行网点是城市中最重要的金融中介, 其 布局是否合理与银行经营效益和民众办理金融业 务的便捷度息息相关。目前,地理学界对银行网点 空间研究多集中于区位选择、空间格局演化及其影 响因素与优化策略等方面。如李翠敏(2005)研究了 上海中心城区不同类型银行的分布, 认为上海银行 机构分布主要受两大类因素影响, 即人口、商务流 量、交通、城市规划等外部因素和银行定位与业务 导向、形象营销所需等内部因素; 邓秀丽(2012)认为 北京银行业的分布表现为核心的高度集中和外围 的零散分布, 行政和交通对其区位分布有较大影 响; 甄茂成等(2013)利用复杂网络理论分析了北京 市中国银行网点的网络特征, 并从中剖析其空间分 布的本质特征。已有成果表明, 国内外对银行业地 理分布的研究日益增多并逐渐深人, 但仍存在一些 缺陷。良好的区位条件是满足银行发展、提升竞争 力的必备条件和重要选址因素, 而良好的区位条件 很大程度上取决于交通的便利程度。交通网络可 达性越好, 越有利于产业集聚, 区位条件也就越 好。但目前学界对银行网点布局与交通之间关系 的研究较为笼统, 理论与实证研究尚未形成体系, 且从城市内部公共交通网络中心性视角分析其与 银行网点分布的空间关联性与耦合性的研究几乎 没有。此外, 已有研究方法多为定性分析, 对交通 网络中心性与社会经济要素的关系研究仅落脚在
线性相关分析上(陈晨等, 2013), 未进一步探索其空 间结构特性。为此, 本文以武汉都市发展区为例, 以城市公共交通网络中最具代表性的公共汽车网 络与地铁网络为研究实体, 基于改进多中心性评价 模型测度公交网络中心性, 运用核密度分析法对公 交网络中心性和银行网点空间分布规律进行分析, 并在此基础上构建公共交通网络中心性与银行网 点分布密度的双变量空间自相关模型, 探究两者的 空间耦合关系, 以期为武汉市银行网点的布局优化 提供理论支撑, 也为城市公共交通可达性及其与社 会活动关系的研究提供一种新思路。

\section{2 数据与方法}

\section{1 研究区域}

武汉市位于中国经济地理中心, 是全国重要的 工业基地、科教基地和综合交通枢纽。长江及其最 大支流汉水横贯武汉市区中央, 将其城区一分为 三,形成了武昌、汉口、汉阳三镇隔江鼎立的空间格 局。本文研究区为《武汉市城市总体规划(20102020年)》所确定的都市发展区, 东到阳逻、双柳、左 岭、豹澥, 西至走马岭、蔡甸城关镇、常福, 北抵天 河、横店、三里, 南达纱帽、金口、郑店和五里界, 是 城市功能的主要集聚区和城市空间的重点拓展 区。由于部分公共交通线路较长, 延伸至都市发展 区界线外(如292 路、301 路), 因此需在都市发展区 基础上,将研究区范围适当扩展至公交线路终点。

\section{2 数据来源与处理}

本文公共交通数据包括公交汽车线路及站点、 地铁线路及站点和道路网。其中, 公交线路及站点 数据来源于武汉公交管理办公室网站(http://www. whjt.gov.cn/gjb/), 时效性强, 能反映武汉公交线路 运营的最新状态。地铁线路及站点数据来自武汉 地铁集团有限公司网站 (http://www.whrt.gov.cn/)。 道路基础数据是基于百度地图、腾讯地图叠加卫星 地图进行配准后矢量化得到的。截至 2015 年 12 月, 研究区内共有 360 条常规公交汽车线路和 4 条 地铁线路,利用 ArcGIS 对各线路及站点进行矢量 化处理,生成武汉市公共交通网络图(图 1)。本文中 银行网点仅指银行实体营业网点, 包括支行、储蓄 所、分理处等分支机构, 不包括 ATM 网点, 相关数 据从中国银行、中国工商银行、中国农业银行、汉口 银行等 23 家银行官方网站上获得。通过百度地图 
找到各银行网点的经纬度坐标, 导人 ArcGIS 形成 矢量数据,进行空间定位与分析。

\section{3 研究方法}

2.3.1 改进多中心性评价模型 (Improved Multiple Centrality Assessment Model)

“单中心”城市空间结构是中国传统的城市发 展模式,大量人口、产业集聚于中心城区,造成了严 重的交通拥堵问题,已成为影响中国城市健康发展 的一大“弊病”(郭研苓, 2011; 孙斌栋等, 2013)。为 缓解单中心蔓延带来的城市问题, 很多大城市纷纷 采取由 “单中心” 向 “多中心”转变的空间发展战 略。《武汉市城市总体规划(2010-2020年)》明确提 出, 要依托主要交通干线, 建成以主城区为核心的 多轴、多中心、开放式的城市空间布局。交通系统 作为城市的骨架, 与城市空间格局相互影响, 一方 面对 “多中心”城市结构具有重要的引导作用; 另一 方面, 交通网络空间结构也会伴随着城市空间格局 的转变而形成多个辐射核心。基于此, 本文以公交 线路、地铁线路及沿实际道路步行换乘线路为边, 公交站点、地铁站点为节点构建网络数据集, 基于 实地调查, 设置常规公交车速为 $20 \mathrm{~km} / \mathrm{h}$, 地铁车速 为 $30 \mathrm{~km} / \mathrm{h}$, 步行速度为 $5 \mathrm{~km} / \mathrm{h}$, 沿着实际网络路径 计算各个节点间的距离与时间成本, 运用改进多中 心性评价模型的接近中心性、介数中心性与直达性 等指标, 测度武汉都市发展区公共交通网络中心 性, 为公交网络与银行网点分布之间的关系进行量 化研究提供基础。

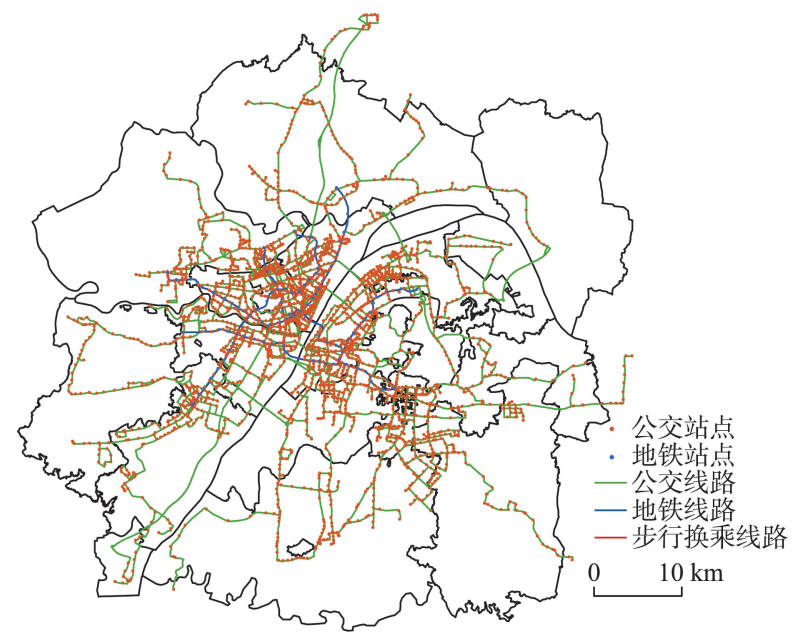

图 1 武汉市公共交通网络示意图

Fig.1 Public transportation network in Wuhan City
(1) 接近中心性

接近中心性表示一个节点与其他所有节点接 近的程度, 公式如下:

$$
C_{i}^{\mathrm{C}}=(N-1) / \sum_{j=1 ; j \neq i}^{N} d_{i j}
$$

式中: $C_{i}^{\mathrm{C}}$ 表示节点 $i$ 的接近中心性; $N$ 为网络节点 数; $d_{i j}$ 表示节点 $i$ 与 $j$ 之间的最短路径。一般意义上 的可达性是指任一点到达目的地的难易程度(李平 华等, 2005), 常以距离、时间为指标来衡量空间阻 力。本文选取的公共交通网络包含距离相同条件 下,时间成本不同的 3 种交通方式,即公共交通网络 数据集的边包含了常规公交线路、地铁线路、站点 步行换乘线路 3 种类型, 对应的实际通行方式与速 度不同。基于各边计算的节点间实际时间存在差 异, 对作为模型参数的最短距离成本路径进行了改 进, 使之更符合当代城市居民高效率、快节奏的生 活出行要求。改进的接近中心性计算公式如下:

$$
C_{i}^{\mathrm{C}}=(N-1) / \sum_{j=1 ; j \neq i}^{N} t_{i j}
$$

式中: $C_{i}^{\mathrm{C}}$ 表示节点 $i$ 的接近中心性; $N$ 表示网络节 点数; $t_{i j}$ 表示节点 $i$ 与 $j$ 之间的最短时间成本。某个 点的接近中心性越大, 则其到网络中任意其他节点 所花费的平均时间就越短, 在网络中也就越趋于中 心位置。因此, 接近中心性反映了节点在网络中的 相对可达性, 并且能从公共交通网络的角度指示城 市结构核心。

\section{(2) 介数中心性}

介数中心性也是一种基于最短距离的度量指 标, 通常被定义为网络中所有节点对的最短路径中 经过节点 $i$ 的最短路径数, 公式如下:

$$
C_{i}^{\mathrm{B}}=\frac{1}{(N-1)(N-2)} \sum_{j=1 ; k=1 ; j \neq k \neq i}^{N} \frac{n_{j k}(i)}{n_{j k}}
$$

式中: $C_{i}^{\mathrm{B}}$ 表示节点 $i$ 的介数中心性; $N$ 表示公共交 通网络节点数; $n_{j k}$ 表示节点 $j$ 与 $k$ 之间的最短路径 数量; $n_{j k}(i)$ 表示节点 $j$ 与 $k$ 之间最短路径中穿过节点 $i$ 的最短路径数量。同理, 本文对该指标稍作修改, 将其定义为网络中所有节点对以最短时间成本连 接的路径中, 经过节点 $i$ 的最短时间成本路径数, 公 式如下:

$$
C_{i}^{\mathrm{B}}=\frac{1}{(N-1)(N-2)} \sum_{j=1 ; k=1 ; j \neq k \neq i}^{N} \frac{m_{j k}(i)}{m_{j k}}
$$

式中: $C_{i}^{\mathrm{B}}$ 为节点 $i$ 的介数中心性; $N$ 为公共交通网 络节点数; $m_{j k}$ 为节点 $j$ 与 $k$ 之间最短时间成本路径 数量; $m_{j k}(i)$ 为节点 $j$ 与 $k$ 之间最短时间成本连接的路 
径中穿过节点 $i$ 的最短时间成本路径数量。式(4)所 表达的实际意义是当穿过某个节点的最短时间成 本路径越多, 介数中心性数值越大。介数中心性可 以反映网络节点上潜在负荷的大小, 运用在公共交 通网络中则衡量各节点承受的交通客流量。

(3) 直达性

公共交通网络基于城市道路又不同于道路网 络, 其在线路设置、站点停靠、行驶方向上存在一定 限制, 站点之间的通行常常无法实现绝对直线化。 在不考虑交通堵塞等情况下, 本文可计算出站点基 于公交网络通行的最短距离, 它既考虑了站点所在 道路的空间特性, 也反映了公共交通因其停靠站 点、线路设置限制造成的绕路等问题的固有特性。 通过测度网络中 2 个节点的最短路径与连接这 2 个 节点的直线路径的偏离程度, 将其作为衡量公共交 通网络效率的指标; 偏离程度越小, 直达性越好, 交 通效率越高。公式为:

$$
C_{i}^{\mathrm{S}}=\frac{1}{N-1} \sum_{j=1 ; j \neq i}^{N} \frac{d_{i j}^{\mathrm{Eucl}}}{d_{i j}}
$$

式中: $C_{i}^{\mathrm{S}}$ 为节点 $i$ 的直达性; $N$ 为网络节点数; $d_{i j}^{\text {Eucl }}$ 为节点 $i$ 与 $j$ 之间的欧氏距离, 即节点 $i$ 与 $j$ 之间的最 短距离。若某一节点能以最短的直线路径到达网 络内任一节点, 说明该节点直达性最佳, 交通效率 也最高。

\subsubsection{GIS 空间密度分析法}

直接用图来表示离散点数据时, 存在难以清晰 地表达其空间分布趋势的弊端。GIS 空间密度分析 法则可通过对离散点数据或线数据进行内插, 从而 得到研究对象空间连续的密度变化图层, 反映其在 整个区域的空间集聚状况。依据插值原理的不同， 可分为核密度分析和普通的点、线密度分析(尹海 伟等, 2014)。核密度分析是通过计算一定窗口范 围内的离散点密度, 将计算结果作为该窗口的中心 值。对于落人搜索范围内的点, 赋予不同的权重, 越接近搜寻中心的点或线权重越大, 反之亦然。因 此, 核密度分析能够反映距离衰减规律。而在一般 的点、线密度分析中, 落在搜索范围内的点或线权 重相同, 无法反映出距离衰减规律(王法辉, 2009)。 接近中心性、介数中心性、直达性等中心性度量指 标是网络节点的属性, 对各中心性指标与其他要素 进行相关分析时需统一分析尺度 (王瑶莉等, 2013)。本文运用ArcGIS 核密度分析工具, 对公共 交通网络中心性与银行网点进行空间插值, 将两者
转换到同一分析单元下, 全面研究武汉都市发展区 内公共交通网络中心性与银行网点的空间分布形 态及规律。

\subsection{3 空间自相关分析}

传统数量统计分析只注重数量值之间的关系, 无法表达其空间关系。空间统计分析则可研究与 地理位置相关的数据间的空间依赖、空间关联或空 间自相关。为更直观地表达研究区内公共交通网 络中心性与银行网点的空间关联模式,本文采用空 间自相关方法来测度两者的空间格局相关性。空 间自相关(Spatial Autocorrelation)是空间统计学的 重要组成部分,包括全局空间自相关和局部空间自 相关两大类。全局空间自相关是对某种现象或属 性值在整个区域的空间特征的描述,检验其在空间 上是否存在聚集、分散或随机等特性。全局自相关 模型计算结果是一个单一数值, 有时会掩盖局部状 态的不稳定性(高爽等, 2011); 而局部空间自相关测 度了研究区域各空间位置与各自周围邻近位置的 同一属性的相关性,能更准确地把握局部空间要素 的集聚与分异特征,从而弥补全局相关性分析的不 足。最常用的局部自相关测度指数为空间关联局 域指标 (Local Indicators of Spatial Association, LI$\mathrm{SA}$ ), 即 Local Moran's $I$ 指数。

本文为探讨银行网点与公共交通网络中心性 之间的空间耦合模式,故采用全局和局域的双变量 空间自相关分析法。首先, 构建双变量全局空间自 相关模型, 通过Moran's $I$ 指数探讨银行网点与公共 交通网络中心性在整个研究区上的相关性。公式 如下:

$$
I=\frac{n \sum_{i=1}^{n} \sum_{j=1}^{n} C_{i j}\left(X_{i}^{a}-\bar{X}_{a}\right)\left(X_{j}^{b}-\bar{X}_{b}\right)}{\sum_{j=1}^{n} \sum_{j=1}^{n} C_{i j} \sum_{i=1}^{n}\left(X_{i}^{a}-\bar{X}_{a}\right)\left(X_{j}^{b}-\bar{X}_{b}\right)}
$$

式中: $n$ 为空间单元总数; $\boldsymbol{C}_{i j}$ 是衡量空间单元 $i 、 j$ 之 间邻接关系的权重矩阵; $X_{i}^{a} 、 X_{j}^{b}$ 分别为空间单元 $i$ 属性 $a$ 的值和空间单元 $j$ 属性 $b$ 的值; $\bar{X}_{a} 、 \bar{X}_{b}$ 分别 为属性 $a 、 b$ 的平均值。 $I$ 取值在 \pm 1 之间,大于 0 表示 空间正相关,越接近 1 , 则正相关性越强; 小于 0 表 示空间负相关, 越接近于 -1 , 则负相关性越强; 等于 0 表示空间无关。

其次, 运用局部双变量空间自相关模型, 以更 好地识别不同空间单位银行网点与公共交通网络 中心性之间的耦合关系。公式如下:

$$
I_{k l}=\frac{X_{k}^{i}-\bar{X}_{k}}{\sigma_{k}} \sum_{j=1}^{n} \boldsymbol{W}_{i j} \frac{\left(X_{l}^{j}-\bar{X}_{l}\right)}{\sigma_{l}}
$$


式中: $I_{k l}$ 为局部双变量空间自相关系数; $X_{k}^{i}$ 为空间 单元 $i$ 属性 $k$ 的值; $X_{l}^{j}$ 为空间单元 $j$ 属性 $l$ 的值; $\bar{X}_{k} 、 \bar{X}_{l}$ 分别为属性 $k 、 l$ 的平均值; $\sigma_{k} 、 \sigma_{l}$ 分别为属 性 $k 、 l$ 的方差; $\boldsymbol{W}_{i j}$ 为空间单元 $i, j$ 之间的空间权重 矩阵。

\section{3 公共交通网络中心性测度及空间分 布特征}

基于改进多中心性评价模型测度接近中心性、 介数中心性、直达性等指标, 运用核密度分析法得 到公共交通网络中心性核密度分布图(图 2a-2c)。 经试验, 接近中心性、介数中心性和直达性核密度 分析的搜索半径分别取 2000、3000、1800 m 为宜, 格 网大小均为 $257.11 \mathrm{~m} \times 257.11 \mathrm{~m}$ 。

\section{1 接近中心性}

由图 2a 可以看出, 公共交通网络接近中心性呈 现出 “核心一边缘一外围” 的空间结构。接近中心 性核密度最高值在 $0.000073416 \sim 0.000091769$ 之间， 位于长江西北的汉口老城区, 大致以解放公园路、 高雄路、中山大道一胜利街、武展西路一游艺路为 界形成了高密度核心区(图 2a中 A 区)。该区域位 于汉口的中心地区, 优良的地理区位和交通条件使 得接近中心性值较大的站点集中在该区域, 如京汉 大道江汉路、香港路苗栗路、解放大道循礼门、新华 路协和医院等公交站, 及“友谊路站” “江汉路站” “香港路站”和换乘站点 “循礼门站”等地铁站, 这些 站点到网络中所有节点的平均时间最短, 在整个公 交网络中处于中心位置。区域 $\mathrm{B}$ 以高密度区域 $\mathrm{A}$ 为核心向外圈层扩散,沿香港路、新华路向西北方 向延伸至康居一路附近, 向南至大夹街附近, 向东 则延伸至解放大道黄浦大街公交站点, 分布范围较 大, 包括解放大道中山公园、京汉大道武胜路、武胜 路泰合广场等公交枢纽, 以及汉口火车站、利济北 路站、青年路站等地铁站点。区域 $\mathrm{C}$ 是位于武昌地 区的次级中心, 分布在以湖北省政府为中心的水果 湖地区, 空间范围较小。区域 $\mathrm{B}$ 和 $\mathrm{C}$ 的接近中心性 核密度值均在 $0.000055062 \sim 0.000073415$ 之间, 区域 内节点到达网络中其他节点的平均时间较短, 接近 中心性较高。

距上述城市中心较远的区域中零星分布着几 个小范围的低核密度区, 如南湖大道以南、关山大 道一大学园路以西、民族大道以东、华师园路以北

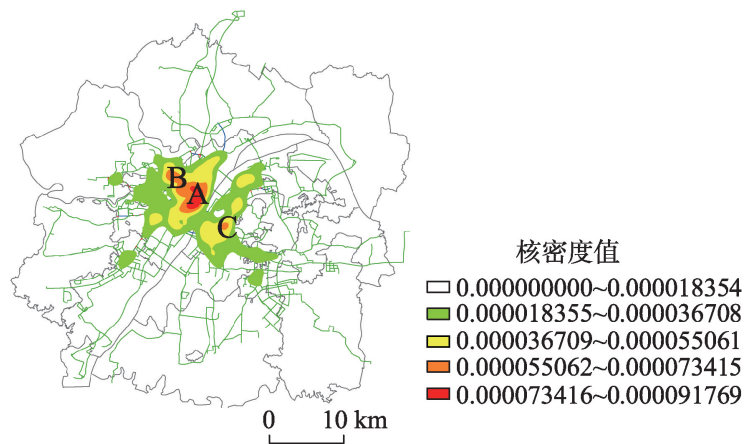

a. 接近中心性

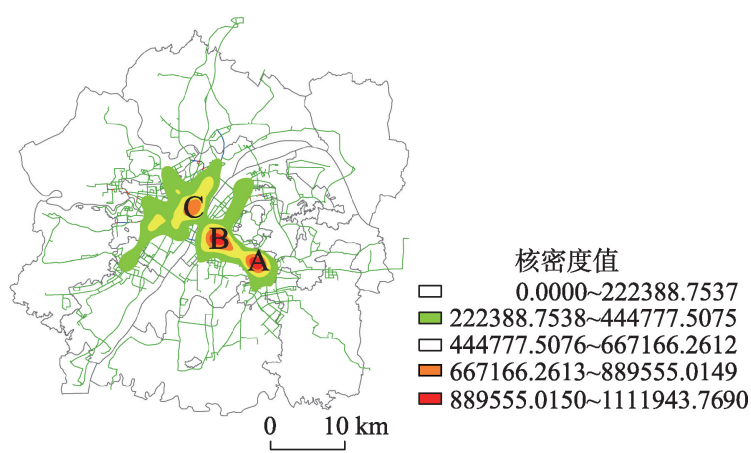

b. 介数中心性

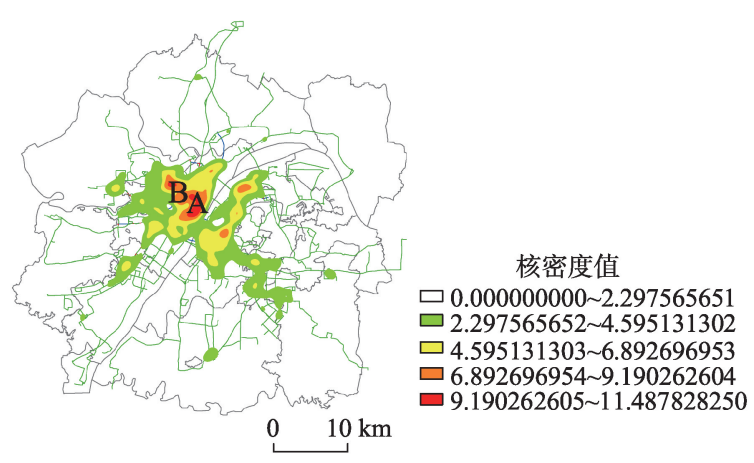

c. 直达性

图 2 武汉公共交通网络中心性核密度分布

Fig.2 Kernel density of public transportation network centralities in Wuhan City

的区域,五环大道、东西湖大道附近, 以及围绕美好 愿景・香榭丽舍、东方花园等住宅小区的神龙大道、 东风大道、联城路、车城北路附近。从武汉三镇的 整体角度看,接近中心性核密度聚集区主要分布于 汉口地区, 其接近中心性明显高于与其隔江相望的 武昌和汉阳地区。可见,汉口地区公共交通站点到 网络所有站点花费的平均时间最少, 在武汉市处于 中心地位。

\section{2 介数中心性}

从图 $2 b$ 可以看出, 公共交通网络介数中心性呈 现出明显的“多中心集聚”结构(图 2b 中的 A、B、C 区), 并呈近似同心圆状分布。核密度最高值在 
889555.0150 1111943.7690 之间, 集中于光谷广场 商圈附近 (图 $2 \mathrm{~b}$ 中 $\mathrm{A}$ 区) 和以中南路地铁换乘站为 中心的中南路、武珞路、中南一路附近(图 $2 b$ 中 B 区), 上述区域内网络节点的介数中心性核密度值 最高。换言之, 网络中所有节点对以最短时间连通 的路径中, 通过该区域内节点的路径数目最多, 说 明该区域内站点在整个公交网络中占据重要地位, 承受的交通客流量最大, 相应的各车站通行压力也 最大, 如民族大道光谷广场、虎泉街地铁光谷广场 站、雄楚大道下钱村等公交站点, 与全市轨道交通 客流量最大、衔接汉口和武昌的地铁 2 号线一期工 程终点站 “光谷广场站” 和地铁 $2 、 4$ 号线的换乘站点 “中南路站”和“洪山广场站”。

区域 $\mathrm{C}$ 是公交网络介数中心性核密度分布的 次级核心区, 大致以京汉大道与江汉路交会处为中 心, 分布在解放大道、京汉大道、中山大道、万松园 路、江汉路等主次干道附近, 包括江汉路地铁循礼 门站、京汉大道大智路、香港路苗栗路等站点。该 区域核密度值处于较高水平,在 667166.2613 889555.0149 之间, 图 2b 中以区域 A、 B 为中心向外 圈层扩散的部分区域的核密度值也在此区间。这 些区域内的公共交通网络介数中心性较高, 表明通 过区域内网络节点的最短时间成本路径较多, 公交 客流相对较大。

纵观全区, 介数中心性核密度呈 $\mathrm{T}$ 字形分布, 以长江一汉江和武珞路一珞喻路为轴向外递减, 形 成了以光谷广场、中南路地铁站、循礼门地铁站为 中心的 3 个高值区。武昌地区的介数中心性核密度 值高于汉口和汉阳, 可见客流量大的公共交通枢纽 多位于武昌地区。

\section{3 直达性}

由图 2c 可知, 直达性核密度分布呈现出 “大分 散、小集聚” 的弱圈层结构, 且布局较分散, 具有城 市多中心指向性特征。直达性核密度最高值在 9.190262605 11.487828250之间, 其覆盖范围内的 公交网络节点到其他任意节点的最短路径与直线 路径偏离程度最小, 交通效率最高。空间上以解放 大道、中山大道、京汉大道、澳门路等交通干线为轴 向两侧扩散,包括香港路站、循礼门站、江汉路站等 地铁站附近区域(图 2c 中 $\mathrm{A}$ 区), 和以长港路站为中 心, 南至新湾路、西至常青路、北至红旗渠路以北、 东至新湾五路的区域。以这 2 个高密度区为中心向 外扩展的区域是直达性较高的集中分布区(图 2c 中
B 区), 核密度值在 6.892696954 9.190262604 之间， 分布范围较广, 大致由江汉路站向西北延伸至常青 花园站。另外,在水果湖广场、光谷商圈、建设二路 与建港南路交叉口、汉阳大道与永丰路路交汇处以 及田园街与四明路交会处附近,形成了小型的中心 一外围圈层分布结构, 这些区域直达性核密度值处 于中上水平。

整体上看, 汉口地区的直达性核密度值平均水 平最高, 且分布范围较集中连片, 武昌地区次之, 汉 阳地区最低。一方面说明总体上汉口地区公共交 通效率最高, 汉阳地区最低; 另一方面, 介数中心性 与直达性及接近中心性的核心匹配度不高。公交 需求大的地方, 公共交通可达性及效率均不高, 说 明武汉市都市发展区内公共交通站点与线路设置 存在不合理性,应在客流量较大的地方增设站点与 线路,协调公交客流与公交可达性及通行效率之间 的关系, 使处于介数中心性核密度集聚区内的交通 站点能快速高效地辐射全区, 避免出现客流拥挤、 交通堵塞等问题。

\section{4 银行网点空间分布特征}

\section{1 总体分布特征}

武汉市都市发展区内 1463 个银行网点的空间 分布如图 3 所示, 其空间格局以贯穿市区的长江和 汉江为界差异显著。汉口地区的银行网点数量最 多, 分布最密集; 其次是武昌区; 汉阳区最少, 仅 98 个。汉口自古被誉为“楚中第一繁盛处”, 以发展金

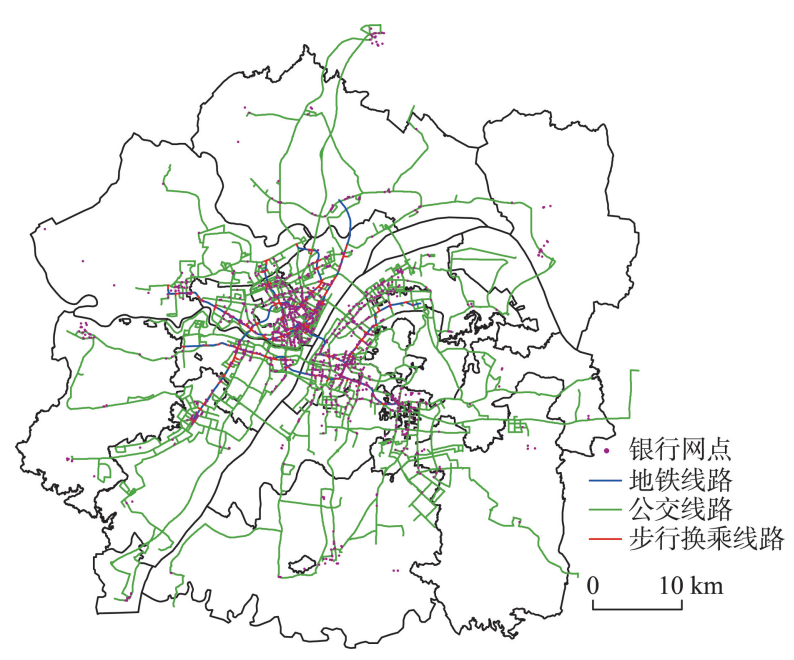

图3 武汉银行网点空间分布

Fig.3 Distribution of bank branches in Wuhan City 
融贸易和商业服务为主要职能; 区内有汉正街传统 商贸区、江汉路商务文化区和堤角都市工业园区， 有中国民生银行大厦、日清洋行大楼等地标建筑， 是外商投资兴业的热土, 吸引着各大金融机构在此 聚集。武昌地区重点发展科教文化、高新技术、金 融商务等职能,建设有中南商务办公区、水果湖行 政办公区、鲁巷城市副中心以及武汉大学、华中科 技大学等众多科研院校, 因而分布着不少银行网 点。汉阳是武汉的工业中心, 以发展制造业为主, 相对而言对银行机构的吸引力较小, 整体上网点布 局较少, 主要集中于钟家村及新兴的王家湾商圈附 近。由此可见, 银行网点数量同城区的发展职能之 间存在一定关系。此外, 本文通过对公交站点建立 $300 \mathrm{~m}$ 缓冲区, 对地铁站点建立 $500 \mathrm{~m}$ 缓冲区, 分别 计算在各自缓冲区范围内银行网点数量, 发现位于 公交站点 $300 \mathrm{~m}$ 缓冲区内的银行网点数达 1277 个, 占银行网点总数的 $87 \%$, 说明银行网点分布与公交 站点服务范围的空间契合度较高。其中, 水果湖路 水果湖、江汉北路新华路口、武珞路丁字桥等公交 站点周边银行网点数量较多, 在 9 12个之间; 而位 于地铁站点 $500 \mathrm{~m}$ 缓冲区内的网点数仅为 409 个, 比例为 $28 \%$, 主要原因是地铁站点总体数量较少, 其服务半径内的银行网点数在整个研究区内所占 比例不高。以银行网点为中心建立的 $300 \mathrm{~m}$ 缓冲区 内有 68 个地铁站点, 占总数的 $71 \%$, 说明银行网点 附近的轨道交通条件较为便利。

\section{2 核密度分布特征}

运用 ArcGIS 的核密度分析工具, 将银行网点 与中心性指标统一到覆盖研究区的等空间分辨率 格网上, 得到银行网点核密度分布图(图 4)。

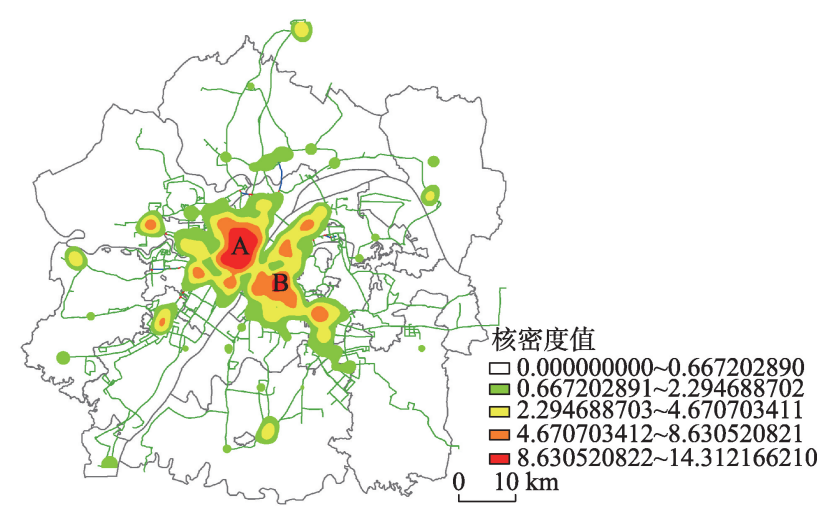

图4 银行网点核密度分布图

Fig.4 Kernel density of bank branches in Wuhan City
图 4 更直观地体现了网点的空间集聚特征, 即 “核心一中心一过渡区一外围”的多核多层次空间 结构。核心区的核密度值最高, 在 8.630520822 14.312166210 之间, 主要集中在汉口内环以江汉路 为代表的中山大道与以武广商圈为核心的解放大 道沿线的“城市商业中心”, 以及被誉为武汉“华尔 街” 的建设大道附近(图 4 中 A 区)。这几条贯通汉 口东西的交通大动脉与新华路一友谊路、台北路、 香港路、澳门路、保成路、利济北路等南北走向城市 主要街道交错形成了较大面积的集聚分布热区。 另一个银行网点布局的高密度核心区为以水果湖 路一姚家岭街、中南路一中北路为横纵轴, 并围绕 洪山广场这一武昌区交通枢纽与商业中心向外扩 散的区域(图 4 中 B 区), 相比之下其范围较小。此 外,还有多个低等级圈层中心分散在核心区外,如 光谷广场商业中心、徐东商圈、钟家村商圈等小型 集聚区。上述集聚区以外的区域,构成了银行网点 的过渡区与外围区。整体上看,武汉市银行网点分 布核密度具有沿江和沿城市主干道发展的特点,武 昌地区无为明显。

\section{5 公共交通网络中心性与银行网点的 空间耦合性}

通过上述分析发现,武汉都市发展区内公共交 通网络中心性和银行网点均存在一定的空间集聚 性。本文借助于 GeoDa 软件, 运用全局和局部的双 变量空间自相关模型,进一步分析公共交通网络中 心性与银行网点之间从整体到局部的空间关联 关系。

\section{1 双变量全局空间自相关}

首先, 运用 ArcGIS 软件提取了研究区内 1463 个银行网点的核密度值, 以及其相同位置处的公共 交通网络中心性核密度值。在 GeoDa 软件中, 以公 共交通网络中心性核密度和银行网点核密度为变 量, 可得到如图 5 所示的公共交通网络接近中心性 一银行网点、介数中心性一银行网点、直达性一银 行网点的Moran 散点图。图中拟合线的斜率即双 变量全局自相关系数 (Moran's $I$ 指数), 分别为 $0.3924 、 0.2918 、 0.3532$, 均为正数, 且图中大部分点 集中在第一、三象限,而第一、三象限分别代表“高 一高”集聚和“低一低”集聚; 第二、四象限分别代表 “低一高”集聚和“高一低”集聚。结果表明,在全局 
空间上,研究区内公共交通网络各中心性指标与银 行网点分布存在着不同的空间关联程度, 均为正相 关。可见, 银行网点分布范围内公共交通条件优 越, 可达性较好, 且各指标与银行网点的空间格局 趋同特征不同。其中, 银行网点核密度与接近中心 性核密度的 Moran's $I$ 指数最大, 空间关联性最强, 意味着银行网点倾向于分布在接近中心性较高的 城市中心, 以获得较好的区位优势, 促进银行更大 限度地获取经营利润。如解放大道以南、江汉路以 西、中山大道以北、民意四路以东的区域以及水果 湖路与中北路交会处, 接近中心性核密度均较高, 社会经济活动密集, 银行网点数量众多且分布集 中, 各银行网点所在位置到公共交通网络中所有节 点花费的平均时间最短, 所能影响的服务区域和对 象分布范围较广。直达性是衡量公共交通效率的 有效指标, 它与接近中心性的核密度空间分布特征 较为相似, 因此直达性与银行网点的核密度空间相 关性亦较强, 可以发现银行网点多分布于公共交通 效率高的区域, 如友谊路、前进路、球场路附近。也
就是说, 通过降低城市居民公共交通的通勤成本、 提高出行效率, 可吸引银行网点布局。

此外, 介数中心性核密度与银行网点核密度之 间也存在显著的空间正相关, 即介数中心性核密度 越高, 银行网点分布越多且越集中。主要原因是介 数中心性数值高低表征了各公交站点与地铁站点 的潜在负荷大小,数值越高的地方, 站点的公共交 通客流量越大, 如光谷广场附近及中南路地铁中南 路站附近等人流密集区, 使银行网点拥有足够的服 务消费人群, 有助于提高银行的经济效益。相比于 接近中心性、直达性, 介数中心性与银行网点的相 关性相对最低, 说明银行网点更倾向于分布在区位 条件好、公共交通效率高的区域。

\section{2 双变量局部空间自相关}

Moran's I 统计量只说明公共交通网络中心性 与银行网点在空间上的平均差异程度,无法反映局 部空间差异。通过 GeoDa 软件的 Multivariate LISA 工具得到武汉都市发展区内 3 个公共交通网络中心 性指标与银行网点布局的 LISA 聚类图(图 6), 进一
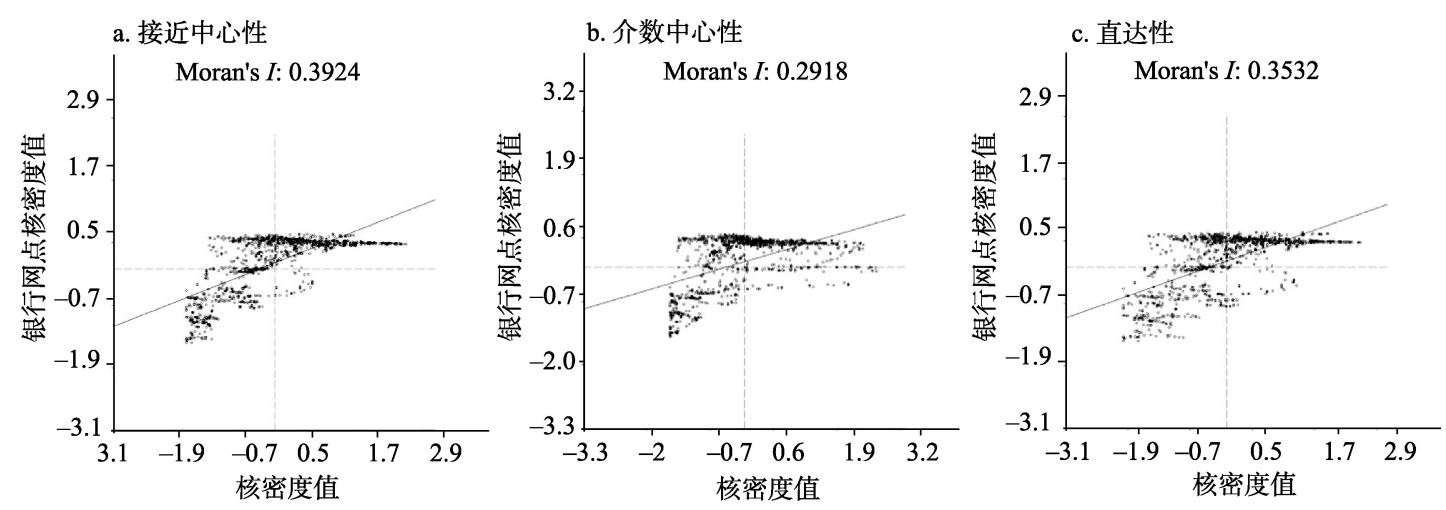

图 5 公共交通网络中心性与银行网点Moran散点图

Fig.5 Moran scatter plot of centrality of public transportation network and bank branches
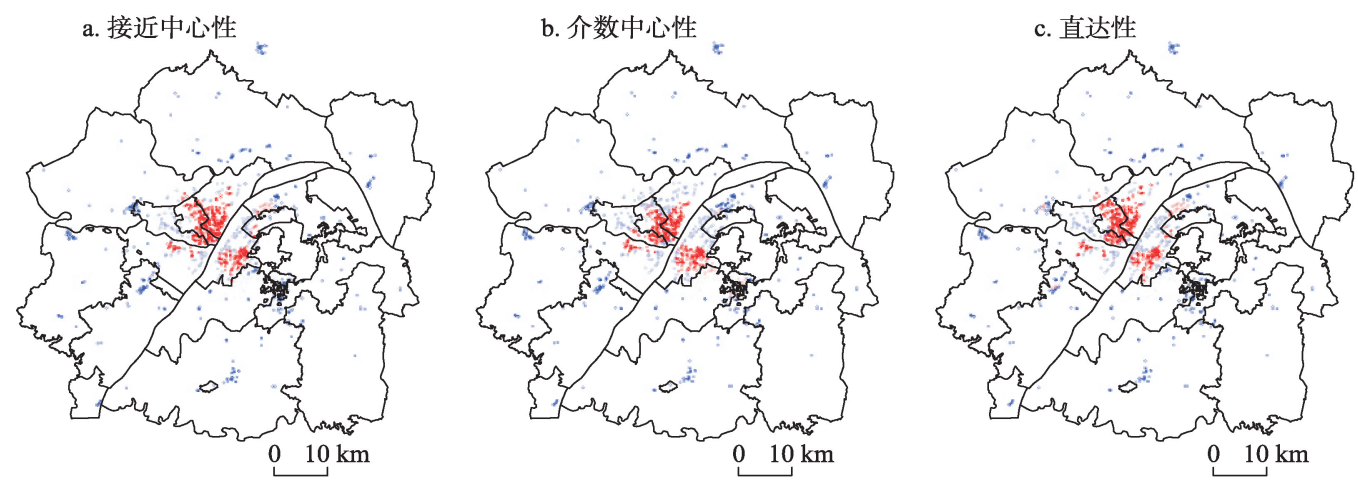

高一高

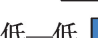

低一高 $\square$ 高一低

图 6 公共交通网络中心性与银行网点的双变量LISA 聚类图

Fig.6 Bivariate LISA cluster map of public transportation network centralities with bank branches 
步挖掘各中心性指标与银行网点的局部聚类特征 信息。

由图 6 可知, 3 个公共交通网络中心性指标与 银行网点的空间耦合均存在 4 种类型的聚集区, 且 表现出高度的空间相似性。其中, 高一高与低一低 2 种类型聚集区构成了公共交通网络中心性与银行 网点分布格局的主要空间关联模式。此外,银行网 点核密度分布的核心区基本属于高一高聚集区,外 围区则基本属于低一低聚集区,即具有银行网点分 布核心圈层的高值指向性与外围圈层的低值指向 性特征,而其他类型聚集区主要位于银行网点核密 度分布的过渡区。

(1) 高一高聚集区。指公共交通网络中心性核 密度值较高, 且银行网点分布密集的区域。该区域 主要分布在解放大道、江汉路附近的汉口内环以及 以中南路、洪山广场为中心的武昌繁华地段, 少部 分位于汉阳区。上述区域均处于武汉市主要建成 区的中心, 集聚了大量商业网点、中小型企业以及 流动人口, 道路交通设施完善, 公共交通网络中心 性与银行网点的分布之间表现出了显著的空间正 相关性。对比图 6a、图 6b、图 6c 可知, 3 个中心性指 标与银行网点之间的耦合关系存在整体相似性的 同时也具有微弱的空间异质性, 即接近中心性与银 行网点空间关联的高一高类型区域范围最大, 直达 性次之,介数中心性最小。

(2) 低一低聚集区。指公共交通网络中心性核 密度值较低, 且银行网点分布少而分散的区域。该 区域也具有正的空间相关性, 主要位于银行网点分 布的外围圈层, 是研究区的边缘地带, 远离热闹喧 器的城市中心, 人口分散, 公共交通条件相对滞后, 站点、路线设置相对较少, 各类社会基础设施与经 济活动强度均不及城市中心地带。对于商业银行 而言,该类区域不具备良好的区位条件, 只需布局 少量银行网点即可满足其周边居民的金融业务 需求。

(3) 低一高聚集区。指公共交通网络中心性核 密度值较低, 但银行网点分布较集中的区域, 主要 位于高一高聚集区与低一低聚集区之间, 部分点缀 于研究区边缘, 具有沿江分布的特点, 如和平大道、 拦江路、古田二路附近。这些区域是银行网点的聚 集热点, 但其公共交通条件却相对欠缺且效率不 高, 可能是因为该区域深厚的历史底蕴及沿江的区 位优势使其发展起步较早, 形成了集商贸、金融、历
史风貌、文化旅游、高档居住等多功能为一体的聚 集区, 吸引了众多银行。而多功能复合使得交通资 源相对稀缺, 易产生交通堵塞, 导致公共交通效率 低下等问题,加之武汉三镇隔江鼎立的空间格局使 得滨江带处于各镇的相对边缘而非中心位置, 沿江 各公交站点的公共交通网络中心性受地形条件限 制及水路交通影响而处于偏低水平。因此,该区域 内银行网点分布与公共交通网络中心性存在非协 调性, 同时说明其公共交通条件有待完善。此外, 部分区域也可能存在银行网点布局过多的问题, 需 对其进行资源整合, 保障资源的利用效率和银行的 服务效益。

（4）高一低聚集区。指公共交通网络中心性核 密度值较高, 但银行网点分布较分散的区域。该空 间关联模式的分布范围很小,但对比图 6a、图 6b、图 $6 \mathrm{c}$ 发现该模式的空间分布差异较大, 图 6a 与图 6c 相似,均分布在和平大道与建设一路、建设四路围 成的区域内; 图 $6 \mathrm{~b}$ 中介数中心性与银行网点的高一 低聚集区则位于关山大道、光谷创业街附近。说明 尽管上述对应区域公共交通站点的接近中心性、直 达性或介数中心性均处于较高水平, 但银行因考虑 到周边地区的设施状况、人口分布与质量状况、经 济发展结构、同行业分布情况、土地租金以及银行 自身经营定位与发展战略等多种内外因素, 并未选 择在此设立网点, 从而产生了公共交通网络中心性 与银行网点的空间非协调性。

\section{6 结论与讨论}

城市公共交通是与城市人民息息相关的民生 工程, 银行网点则是城市中最重要的金融中介, 两 者均是城市地理、城市规划研究中的重要课题。本 文基于改进多中心性评价模型和双变量空间自相 关模型, 对武汉都市发展区公共交通网络中心性, 以及其与银行网点布局的空间耦合性进行了定量 研究, 有助于把握武汉市公共交通网络中心性的空 间分布状态, 为科学规划城市公共交通建设以及合 理布局银行网点提供参考。主要结论有以下几点:

(1) 武汉都市发展区公共交通网络中心性具有 城市多中心指向性特征, 且由中心向外围圈层递 减。位于汉口中心地带的京汉大道江汉路、“江汉 路站”、“循礼门站”等站点的接近度中心性最高, 处 于城市中心位置; 光谷广场附近区域站点的介数中 
心性最高, 交通客流量与通行压力最大, 为城市另 一中心; 解放大道、中山大道沿线的香港路站、江汉 路站等站点直达性最高, 公共交通效率最好, 也是 城市中心之一。

(2) 武汉公共交通网络空间结构已形成多个辐 射核心, 正处于一个良性的发展状态, 但同时需避 免中心区交通负荷过重等问题。如 “光谷广场” 附 近人员密集, 客流量巨大, 地铁、公交等现有路线、 车次仍难以满足大众的出行需求。因此, 在未来城 市公交网络规划与建设中, 应加强“疏”与 “导”。 “疏”在于继续提升中心地区公交网络的服务能力, “导”在于引导和加强非中心地区的公交网络建设, 促进城市交通结构的多中心化的协调发展。

(3) 武汉都市发展区内银行网点分布呈现“核 心一中心一过渡区一外围” 多核多层次的空间结 构, 且区域差异明显。核心区域在汉口内环的中山 大道、解放大道和建设大道等主干道与武昌的洪山 广场周围。

(4) 整体上看, 公共交通网络各中心性指标与 银行网点之间具有良好的空间正相关性, 但关联程 度存在差异。银行网点倾向于分布在接近中心性 较高、区位条件较好的城市中心, 公共交通效率高 的地方也颇受银行青睐。但从局部空间角度分析, 银行网点分布与公共交通网络耦合关系具有空间 不平稳性和空间异质性, 以高一高聚集与低一低聚 集为主, 但还存在高一低聚集和低一高聚集这 2 种 偏离一般规律的现象。高一高聚集位于银行网点 布局的核心圈层，低一低聚集则点缀于外围圈层; 高一低聚集与低一高聚集零星分布于核心圈层与 外围圈层之间。这一方面说明银行网点的分布还 受其他多重因素, 如人口流动、经济活动、同行业分 布情况、政策引导及银行自身发展需要等的影响; 另一方面, 也反映出目前部分区域存在银行网点布 局不合理的情况, 如在低一高聚集区内银行网点布 局过于集中, 但其公共交通条件不佳, 一定程度上 影响了市民办理金融业务的便捷程度, 不利于地区 金融服务业的发展。因此, 银行网点布局时应整合 资源, 合理布局, 实现效益最优化发展。

当然, 本文尚存在一些不足之处, 如在研究公 共交通网络中心性时并未考虑常规公交的站点停 靠时间,且不同时段内不同公共交通线路的通行能 力有较大差异, 从而影响其运行速度和时间。不同 等级与性质的银行网点与公共交通网络中心性间
的相关性也存在差异, 而本文并未对银行网点的等 级及所属银行性质进行细分。在未来的研究中, 应 针对这些不足开展进一步探讨。

\section{参考文献(References)}

曹小曙, 杨文越, 黄晓燕. 2015. 基于智慧交通的可达性与交 通出行碳排放: 理论与实证 [J]. 地理科学进展, 34(4): 418-429. [Cao X S, Yang W Y, Huang X Y. 2015. Aaccessibility and $\mathrm{CO}_{2}$ emissions from travel of smart transportation: Theory and empirical studies[J]. Progress in Geography, 34(4): 418-429.]

陈晨, 程林, 修春亮. 2013. 沈阳市中心城区交通网络中心性 及其与第三产业经济密度空间分布的关系 [J]. 地理科学 进展, 32(11): 1612-1621. [Chen C, Cheng L, Xiu C L. 2013. Distribution of centrality of traffic network and its relationship with economic density of tertiary industry in Shenyang[J]. Progress in Geography, 32(11): 1612-1621.]

程钰, 刘雷, 任建兰, 等. 2013. 济南都市圈交通可达性与经 济发展水平测度及空间格局研究 [J]. 经济地理, 33(3): 59-64. [Cheng Y, Liu L, Ren J L, et al. 2013. The study about measuring of the relationship between transportation accessibility and the level of economic growth and spatial structure at county level[J]. Economic Geography, 33(3): 59-64.]

邓秀丽. 2012. 北京市金融服务业空间格局及其演变研究 [D]. 北京: 首都师范大学. [Deng X L. 2012. Beijingshi jinrong fuwuye kongjian geju jiqi yanbian yanjiu[D]. Beijing, China: Capital Normal University.]

高爽, 魏也华, 陈雯, 等. 2011. 发达地区制造业集聚和水污 染的空间关联: 以无锡市区为例 [J]. 地理研究, 30(5): 902-912. [Gao S, Wei Y H, Chen W, et al. 2011. Study on spacial-correlation between water pollution and industrial agglomeration in the developed region of China: A case study of Wuxi City[J]. Geographical Research, 30(5): 902912.]

高自友, 吴建军, 毛保华, 等. 2005. 交通运输网络复杂性及 其相关问题的研究 [J]. 交通运输系统工程与信息, 5(2): 79-84. [Gao Z Y, Wu J J, Mao B H, et al. 2005. Study on the complexity of traffic networks and related problems[J]. Journal of Transportation Systems Engineering and Information Technology, 5(2): 79-84.]

郭研苓. 2011. 多中心空间结构对城市交通的影响研究: 以 上海为例 $[D]$. 上海: 华东师范大学. [Guo Y L. 2011. Research on the influence of poly-centric spatial impacting on the urban transport: A case of Shanghai[D]. Shanghai, China: East China Normal University.]

黄文雄, 代筠杰, 谭利英, 等. 2009. 基于 “两型社会” 的武汉 中心城区交通问题研究[J]. 华中科技大学学报: 城市科 
学版, 26(4): 62-66. [Huang W X, Dai Y J, Tan L Y, et al. 2009. Traffic problems of Wuhan City center based on constructing resource-conserving and environmentally friendly society[J]. Journal of Huazhong University of Science and Technology: Urban Science Edition, 26(4): 62-66.] 李翠敏. 2005. 城市银行网点的空间分析: 以上海市中心城 区为例 $[D]$. 上海: 上海师范大学. [Li C M. 2005. Chengshi yinhang wangdian de kongjian fenxi: Yi Shanghaishi zhongxin chengqu weili[D]. Shanghai, China: Shanghai Normal University.]

李刚. 2014. 基于网络中心性的城市轨道交通应急救援站选 址研究[D]. 北京: 北京交通大学. [Li G. 2014. Research on location of urban rail transit emergency rescue stations based on network centrality[D]. Beijing, China: Beijing Jiaotong University.]

李立, 汪德根. 2012. 城市低碳公共交通对旅游景点通达性 影响研究: 以苏州市为例 [J]. 经济地理, 32(3): 166-172. [Li L, Wang D G. 2012. The impact of urban low-carbon public transport to tourist attractions' accessibility: Suzhou City area as the example[J]. Economic Geography, 32(3): 166-172.]

李平华, 陆玉麒. 2005. 可达性研究的回顾与展望 $[\mathrm{J}]$. 地理科 学进展, 24(3): 69-78. [Li P H, Lu Y Q. 2005. Review and prospectation of accessibility research[J]. Progress in Geography, 24(3): 69-78.]

李清泉, 萧世伦, 方志祥, 等. 2012. 交通地理信息系统技术 与前沿发展 $[\mathrm{M}$ ]. 北京: 科学出版社. [Li Q Q, Xiao S L, Fang Z X, et al. 2012. Jiaotong dili xinxi xitong jishu yu qianyan fazhan[M]. Beijing, China: Science Press.]

李涛, 曹小曙, 黄晓燕. 2012. 珠江三角洲交通通达性空间格 局与人口变化关系 [J]. 地理研究, 31(9): 1661-1672. [Li T, Cao X S, Huang X Y. 2012. The relationship between spatial structure of accessibility and population change in Pearl River Delta[J]. Geographical Research, 31(9): 16611672.]

李志, 周生路, 吴绍华, 等. 2014. 南京地铁对城市公共交通 网络通达性的影响及地价增值响应 [J]. 地理学报, 69(2): 255-267. [Li Z, Zhou S L, Wu S H, et al. 2014. The impact of metro lines on public transit accessibility and land value capture in Nanjing[J]. Acta Geographica Sinica, 69(2): 255-267.

孙斌栋, 涂婷, 石巍, 等. 2013. 特大城市多中心空间结构的 交通绩效检验: 上海案例研究 [J]. 城市规划学刊, (2): 6369. [Sun B D, Tu T, Shi W, et al. 2013. Test on the performance of polycentric spatial structure as a measure of congestion reduction in megacities: The case study of Shanghai[J]. Urban Planning Forum, (2): 63-69.]

田关云. 2014. 基于 TransCAD 的城市公共交通网络可达性
研究 [D]. 兰州: 兰州交通大学. [Tian G Y. 2014. Studies on the urban public transit network accessibility based on TransCAD[D]. Lanzhou, China: Lanzhou Jiaotong University.]

王法辉. 2009. 基于 GIS 的数量方法与应用[M]. 姜世国, 滕骏 华, 译. 北京: 商务印书馆. [Wang F H. 2009. Jiyu GIS de shuliang fangfa yu yingyong[M]. Jiang $\mathrm{S} \mathrm{G}$, Teng J H, Trans.. Beijing, China: Business Press.]

王薇, 吴丹. 2012. 武汉市优先发展城市公共交通的思考 [J]. 科技致富向导, (18): 23-24. [Wang W, Wu D. 2012. Wuhanshi youxian fazhan chengshi gonggong jiaotong de sikao[J]. Guide of Sci-tech Magazine, (18): 23-24.]

王瑶莉, 高松, 刘瑜. 2013. 青岛城市道路邻近中心性及其应 用方法 [J]. 地理研究, 32(3): 452-464. [Wang Y L, Gao S, Liu Y. 2013. Exploration into urban street closeness centrality and its application methods: A case study of Qingdao [J]. Geographical Research, 32(3): 452-464.]

王喆, 彭其渊. 2007. 成都市公交复杂网络拓扑特性研究 [J]. 交通与计算机, 25(2): 39-42. [Wang Z, Peng Q Y. 2007. Research on topologic properties of Chengdu public traffic complex network[J]. Computer and Communications, 25 (2): 39-42.]

徐黔予. 2011. 优先发展城市公共交通的意义与策略 [J]. 决 策咨询, (2): 71-73. [Xu Q Y. 2011. The meaning and strategy of priority to the development of urban public transport [J]. Decision-Making \& Consultancy, (2): 71-73.]

许文娟. 2014. 城市公共交通与城市发展的适应性评价研究 [D]. 北京: 北京交通大学. [Xu W J. 2014. Study on adaptability evaluation between public transport and urban development[D]. Beijing, China: Beijing Jiaotong University.]

尹海伟, 孔繁花. 2014. 城市与区域规划空间分析实验教程 [M]. 南京: 东南大学出版社. [Yin H W, Kong F H. 2014. Lab manual for spatial analysis in urban and regional planning[M]. Nanjing, China: Southeast University Press.]

赵莉莉. 2010. 复杂网络理论在城市公交系统中的应用[D]. 石家庄: 河北师范大学. [Zhao L L. 2010. The application of complex networks in urban bus transport systems[D]. Shijiazhuang, China: Hebei Normal University.]

甄茂成, 张景秋, 杨广林. 2013. 基于复杂网络的商业银行网 点布局特征: 以北京市中国银行为例 $[\mathrm{J}]$. 地理科学进展, 32(12): 1732-1741. [Zhen M C, Zhang J Q, Yang G L. 2013. Characteristics of commercial bank branch networks based on complex networks theory: A case study on bank of China in Beijing $[\mathrm{J}]$. Progress in Geography, 32(12): 1732-1741.]

Bryson J R. 1997. Business service firms, service space and the management of change[J]. Entrepreneurship \& Region- 
al Development, 9(2): 93-112.

Latora V, Marchiori M. 2002. Is the Boston subway a smallworld network[J]. Physica A: Statistical Mechanics and Its Applications, 314(1-4): 109-113.
Sienkiewicz J, Holyst J A. 2005. Public transport systems in Poland: From Bialystok to Zielona Góra by bus and tram using universal statistics of complex networks[J]. Acta Physica Polonica B, 36(5): 1771-1778.

\title{
Centrality of public transportation network and its coupling with bank branches distribution in Wuhan City
}

\author{
ZHAN Xuan ${ }^{1,2}$, LIN Aiwen ${ }^{1,2^{*}}$, SUN Cheng ${ }^{1,2}$, QIAO Wei ${ }^{1}$ \\ (1. School of Resource and Environment Science, Wuhan University, Wuhan 430079, China; \\ 2. Key Laboratory of Geographic Information System, Ministry of Education, Wuhan 430079, China)
}

\begin{abstract}
Urban public transportation is an indispensable part of urban life and an important topic in today's urban geography research. Existing studies on public transportation mainly focused on the characteristics of network structure and topology. Centrality is one of the important properties of public transportation network, and is widely examined both in theoretical and empirical studies. Centrality can be effectively calibrated by Multiple Centrality Assessment Model (MCA), which is composed of multiple measures such as closeness, betweenness, and straightness. In recent years, researchers began to focus increasingly more on the study of the relationship between public transportation network and social and economic activities. As the most important financial intermediary in the city, banks and the distribution of their branches are closely related to the efficiency of people's financial activities. Thus, we investigate the spatial pattern of public transportation network centrality and its coupling with bank branches in Wuhan City. First, this study builds a dataset consisting of public transportation and bank branches in Wuhan urban development zone of 2015 in ArcGIS. Based on the characteristics of the data, this study improves the Multiple Centrality Assessment Model for better accuracy. It then examines the geography of three centrality indices by improved Multiple Centrality Assessment Model, and analyzes the centrality of Wuhan public transportation network and its spatial correlation with bank network layout by using kernel density estimation and bivariate spatial autocorrelation model based on GeoDa. The results show that: (1) Kernel density indicates a clear city multicenter directivity of the public transportation network centralities in Wuhan, and the concentration decreases progressively from the central areas to the periphery. (2) The spatial distribution pattern of bank branches presents a core - center - transitional areaperiphery multi- level structure, and the regional variations between different areas are obvious. (3) There is a significant positive correlation between public transportation network centralities and the bank network layout in Wuhan City. Among the three centrality indicators, closeness has the greatest effect on the distribution of bank branches, straightness comes the next, and betweenness has minimum impact on bank branches' distribution. (4) At the local level, spatial correlation patterns between public transportation network centralities and bank branches exhibit spatial nonstationarity and heterogeneity. The local spatial correlation dendrogram indicates that "HighHigh" and "Low-Low" coupling are the main spatial correlation patterns. The distribution characteristics of the "High-High" type cluster to the central area, and "Low-Low" type cluster in the peripheral area, are prevalent. "High-Low" and "Low-High" types are distributed sporadically in the transitional areas. This result also indicates that the distribution of bank branches is affected by various factors, including public transportation network centralities.
\end{abstract}

Key words: centrality of public transportation network; Improved Multiple Centrality Assessment Model; bank branches; coupling; Wuhan urban development zone 